CRYSTALLOGRAPHIC COMMUNICATIONS

ISSN 2056-9890

Received 21 November 2017

Accepted 19 December 2017

Edited by W. T. A. Harrison, University of Aberdeen, Scotland

Keywords: crystal structure; fluorescein; hydrogen bonding; $\mathrm{Cl} \cdots \pi$ interaction.

CCDC reference: 1812489

Supporting information: this article has supporting information at journals.iucr.org/e

\section{Crystal structure of $4^{\prime}$-allyl-4,5,6,7,2', $7^{\prime}$-hexa- chlorofluorescein allyl ester unknown solvate}

\author{
Lili Wang, Alexander S. Filatov and Gregory S. Engel*
}

Department of Chemistry, James Franck Institute, Institute for Biophysical Dynamics, University of Chicago, 5735 South Ellis ave, Chicago, IL 60637, USA. *Correspondence e-mail: gsengel@uchicago.edu

In the title compound, $4^{\prime}$-allyl-4,5,6,7,2,, $7^{\prime}$-hexachlorofluorescein allyl ester \{systematic name: prop-2-en-1-yl 2,3,4,5-tetrachloro-6-[2,7-dichloro-6-hydroxy3-oxo-4-(prop-2-en-1-yl)-3H-xanthen-9-yl]benzoate\}, $\mathrm{C}_{26} \mathrm{H}_{14} \mathrm{Cl}_{6} \mathrm{O}_{5}$, accompanied by unknown solvate molecules, the dihedral angle between the xanthene ring system (r.m.s. deviation $=0.046 \AA$ ) and the pentasubstituted benzene ring is $71.67(9)^{\circ}$. Both allyl groups are disordered over two sets of sites in statistical ratios. The scattering contributions of the disordered solvent molecules (both $\mathrm{Ph}_{2} \mathrm{O}$ and $\mathrm{CHCl}_{3}$, as identified by NMR) were removed with the PLATON SQUEEZE algorithm [Spek (2015). Acta Cryst. C71, 9-18]. In the crystal, tetrameric supramolecular aggregates linked by $\mathrm{O}-\mathrm{H} \cdots \mathrm{O}$ hydrogen bonds occur; these further interact with neighboring aggregates through $\mathrm{C}-\mathrm{Cl} \cdots \pi$ interactions arising from the benzene rings, forming infinite two-dimensional sheets. Each $\mathrm{C}_{6} \mathrm{Cl}_{4}$ ring shifts in the direction perpendicular to the twodimensional sheet, exhibiting a helical chain in which every $\mathrm{C}_{6} \mathrm{Cl}_{4}$ ring is utilized as both a donor and an acceptor of $\mathrm{Cl} \cdots \pi$ contacts. Thus, these two-dimensional sheets pack in a helical fashion, constructing a three-dimensional network.

\section{Chemical context}

Fluorescein derivatives have been widely used in chemical and biological research. The high fluorescence quantum yields and excellent photostability of these dyes make them attractive as fluorescent labels for macromolecules such as proteins (Giepmans et al., 2006) or DNA (Li et al., 1995). Fluorescein derivatives also exhibit tunable optical transitions in the visible range and high molar extinction coefficients, making them suitable for optical laser and dye-sensitized solar cell applications (Pepe et al., 2016). Understanding the properties of these fluorescein derivatives, especially their bonding abilities at certain local environments, is essential for designing and utilizing these compounds. Detailed crystal structure determinations of fluorescein derivatives can reveal their bonding/packing properties, providing valuable insights in directing future molecular engineering design and chemical and biological applications. Until recently, the different forms of fluorescein could only be obtained as microcrystalline powders and the first crystal structure determination of free fluorescein came from powder diffraction data analysis (Tremayne et al., 1997). It was then followed by a number of single crystal X-ray structural analyses of fluorescein derivatives. For several recent examples, see Christianson \& Gabbaï (2016), Sezukuri et al. (2016), and Dufresne et al. (2007).

The title compound, 4'-allyl-4,5,6,7,2',7'-hexachlorofluorescein allyl ester, is an important intermediate in the 
synthetic route of structurally flexible fluorescein heterodimers that were recently published by us (Wang et al., 2017). Such heterodimers were designed to test the engineering principle of quantum coherences in artificial light-harvesting systems. Herein, we present the crystal structure of the title compound, which reveals the importance of $\mathrm{Cl} \cdots \pi$ interactions in the solid state.<smiles>C=C=C=C</smiles>

(A)

(I)<smiles>C=CCOC(=O)c1c(Cl)c(Cl)c(Cl)c(Cl)c1-c1c2cc(Cl)c(=O)cc-2oc2c(CC=C)c(O)c(Cl)cc12</smiles>

(B)

\section{Structural commentary}

The molecular structure of the title compound is shown in Fig. 1. The structure consists of a xanthene ring system, a perchlorinated phenyl ring and two allyl groups; one is located

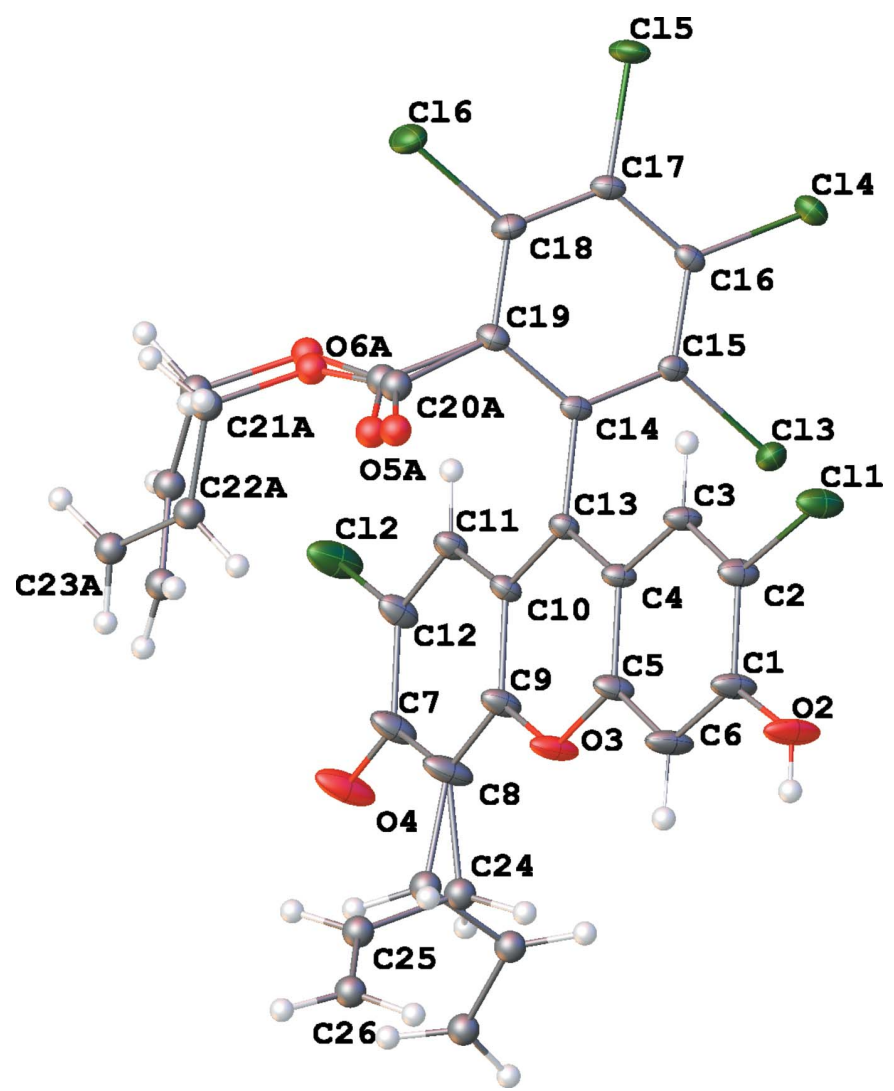

Figure 1

The molecular structure of the title compound with $40 \%$ displacement ellipsoids. $\mathrm{H}$ atoms as well as atoms of the disordered allyl groups are shown as spheres of arbitrary radius.
Table 1

Hydrogen-bond geometry $\left(\AA,^{\circ}\right)$.

\begin{tabular}{lllll}
\hline$D-\mathrm{H} \cdots A$ & $D-\mathrm{H}$ & $\mathrm{H} \cdots A$ & $D \cdots A$ & $D-\mathrm{H} \cdots A$ \\
\hline $\mathrm{O} 2-\mathrm{H} 2 \cdots \mathrm{O} 4{ }^{\mathrm{i}}$ & $0.74(4)$ & $1.86(4)$ & $2.595(3)$ & $172(4)$ \\
\hline
\end{tabular}

Symmetry code: (i) $y+\frac{1}{4},-x+\frac{3}{4},-z+\frac{3}{4}$.

at the periphery of the xanthene ring while the other is linked to the six-membered ring through the carboxylate linker (atom O6). The phenyl plane inclines from the xanthene plane by about $73^{\circ}$ [the $\mathrm{C} 4-\mathrm{C} 13-\mathrm{C} 14-\mathrm{C} 15$ torsion angle is $\left.72.7(3)^{\circ}\right]$. The unusual unsymmetrical substitution pattern on the xanthene ring of the title compound leads to the possibility of having different tautomers as depicted in the Scheme. Unsymmetrically substituted fluoresceins have previously been reported, but until now all related structural reports showed only their spiroxanthene isomeric forms (Hou et al., 2012; Swamy et al., 2006; Wang et al., 2005), thwarting a direct comparison with this study. While the title compound may exist as a mixture of exchanging tautomers $(A)$ and $(B)$ in solution, the solid-state structure is better described as tautomer $(A)$ based on the bond-length distribution. For example, the bond lengths for C7-O4 [1.251 (3) $\AA$ ] and $\mathrm{C} 1-$ $\mathrm{O} 2[1.326(3) \AA]$ are consistent with a $\mathrm{C}=\mathrm{O}$ double bond and a $\mathrm{C}-\mathrm{O}$ single bond, respectively. The bond lengths of $\mathrm{C} 8-\mathrm{C} 9$, $\mathrm{C} 10-\mathrm{C} 13$, and $\mathrm{C} 11-\mathrm{C} 12$, which are 1.359 (4), 1.373 (3), and 1.347 (3) $\mathrm{A}$, respectively, are significantly shorter than C7$\mathrm{C} 12$ and $\mathrm{C} 10-\mathrm{C} 11$ [1.459 (4) and 1.429 (3) А, respectively], suggesting that the former are of a double-bond character. It should be noted here that this tautomer may not represent the

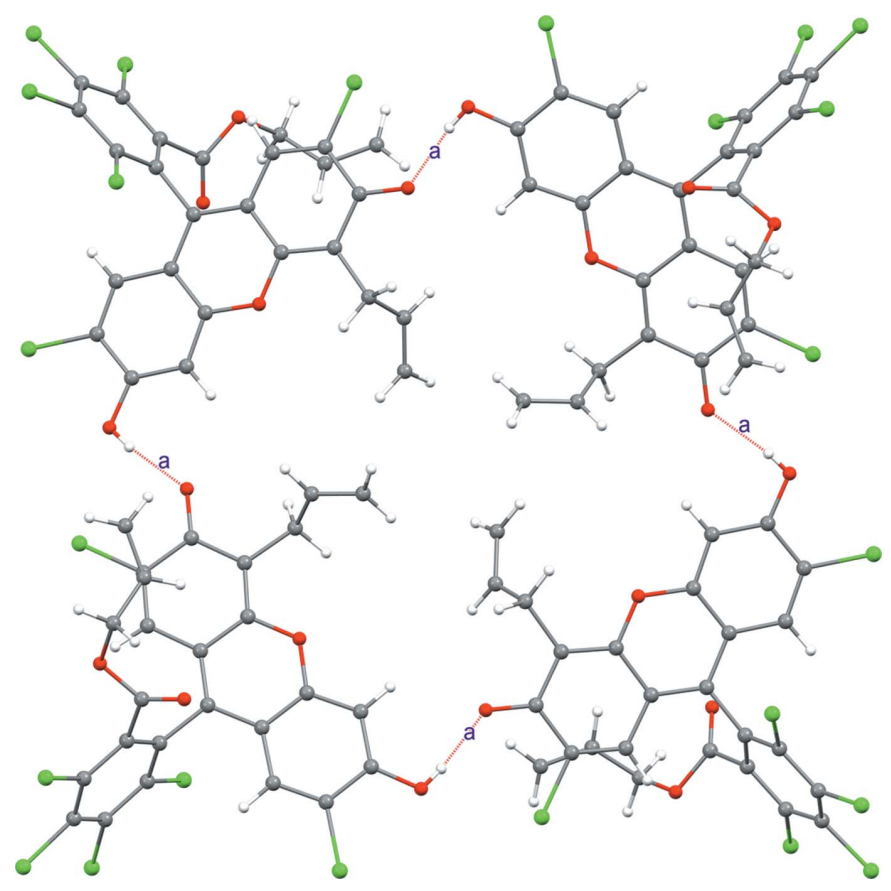

Figure 2

A tetrameric hydrogen-bonded aggregate formed by the title compound: $\mathrm{O} 2-\mathrm{H} 2 \cdots \mathrm{O} 4$ bonds are labeled as ' $\mathrm{a}$ '. The assemblage has $\overline{4}$ symmetry. 


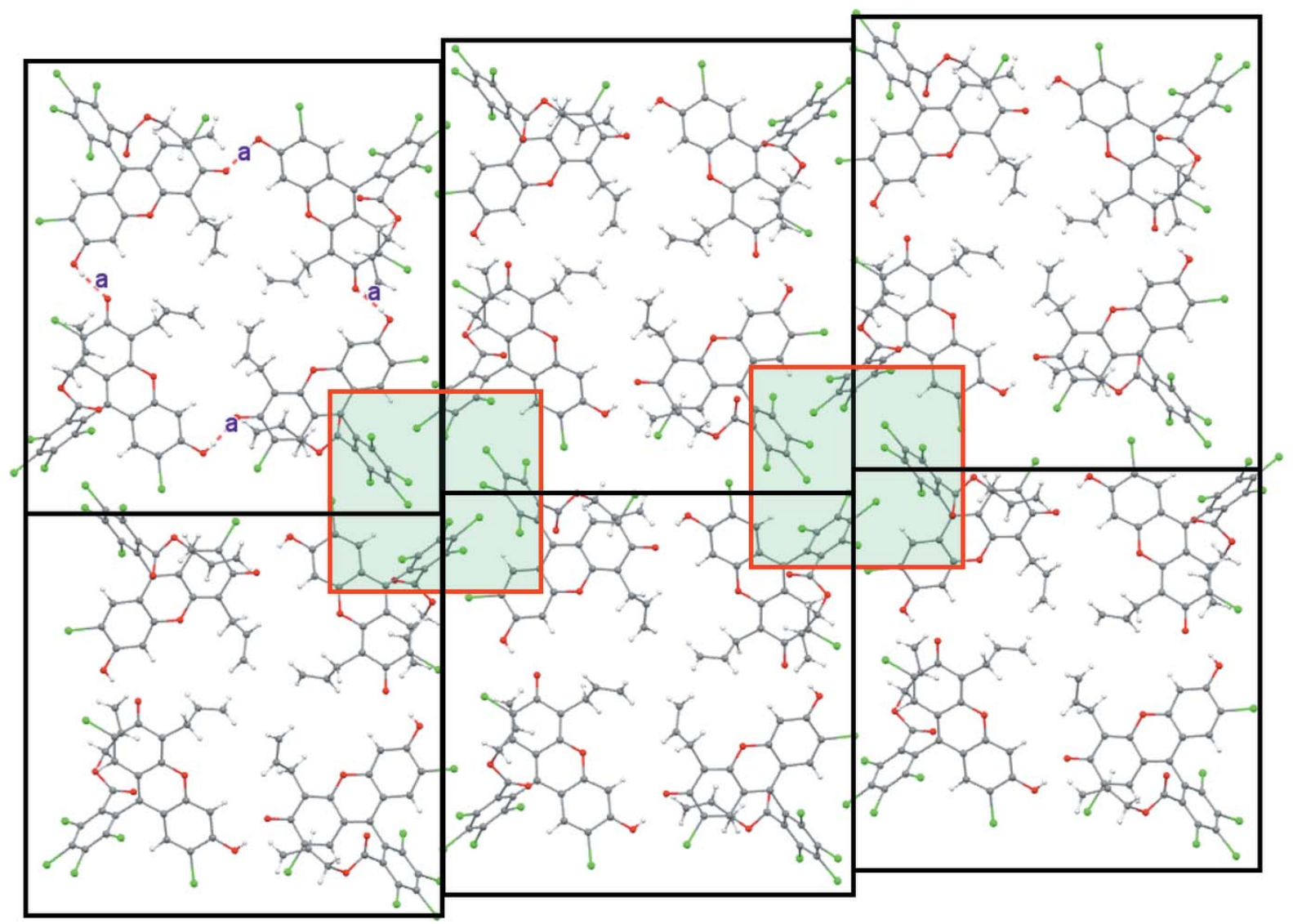

Figure 3

Infinite sheets formed by neighboring tetrameric aggregates via $\mathrm{Cl} \cdots \pi$ interactions. The aggregates are shown as large black squares and the intermolecular interactions between them are shown as small red squares with a semi-transparent green background.

thermodynamically more stable tautomer that may exist in the gas phase, because this form may be stabilized by the formation of tetrameric aggregates through intermolecular $\mathrm{O} 2-$ $\mathrm{H} 2 \cdots \mathrm{O} 4$ bonds as discussed below (Table 1, Fig. 2).

\section{Supramolecular features}

In the crystal, the title compound forms tetrameric aggregates linked by $\mathrm{O} 2-\mathrm{H} 2 \cdots \mathrm{O} 4$ hydrogen bonds, as shown in Fig. 2. The allyl groups sit inside the pocket formed by the hydrogen bonds and are not engaged in any particular intermolecular interactions (only one disorder component is shown). The tetrameric aggregates further interact with neighboring aggregates through $\mathrm{Cl} \cdots \pi$ interactions of dangling $\mathrm{C}_{6} \mathrm{Cl}_{4}$ rings forming infinite two-dimensional sheets, as shown in Fig. 3. Each of the $\mathrm{C}_{6} \mathrm{Cl}_{4}$ rings accepts two edge-on $\mathrm{Cl} \cdots \mathrm{C}$ short contacts from an adjacent $\mathrm{C}_{6} \mathrm{Cl}_{4}$ unit $[\mathrm{Cl} 4 \cdots \mathrm{C} 16=3.398$ (3); $\mathrm{Cl} 5 \cdots \mathrm{C} 18=3.333(3) \AA]$. When viewed along the twodimensional sheet located in the $a b$ plane, it may be noted that each $-\mathrm{C}_{6} \mathrm{Cl}_{4}$ ring is in fact shifted in the direction perpendicular to the two-dimensional sheet. These $\mathrm{C}_{6} \mathrm{Cl}_{4}$ rings thus exhibit a helical chain in which every $\mathrm{C}_{6} \mathrm{Cl}_{4}$ ring is utilized as both a donor and an acceptor of $\mathrm{Cl} \cdots \pi$ contacts. Thus, several layers of the tetrameric aggregates are further packed in a helical manner in the third dimension along the $c$ axis, constructing a three-dimensional network, as shown in Fig. 4.

\section{Database survey}

A search of the Cambridge Structural Database (CSD, Version 5.38, update May 2017; Groom et al., 2016) indicated that several fluorescein derivatives with halogen substituents on the xanthene ring have been reported (Cody, 1987; Willner et al., 1992; Harrison et al., 2007; Quint et al., 2016). However, there was only one structural report on fluorescein derivatives that contains a tetrachloro-substituted phenyl unit (CCDC refcode KUFTUA; Willner et al., 1992), and there were no structural reports on hexachlorinated fluorescein derivatives. While the hydroxyl groups on the xanthene rings of fluorescein derivatives have been reported to engage in hydrogen bonds (Abrahams et al., 2009), to the best of our knowledge, the tetrameric aggregation motif in this report has not been found previously for fluorescein derivatives.

\section{Synthesis and crystallization}

$4,5,6,7,2^{\prime}, 7^{\prime}$-Hexachlorofluorescein diallyl ether ester was synthesized following a literature method (Wang et al., 2017). 


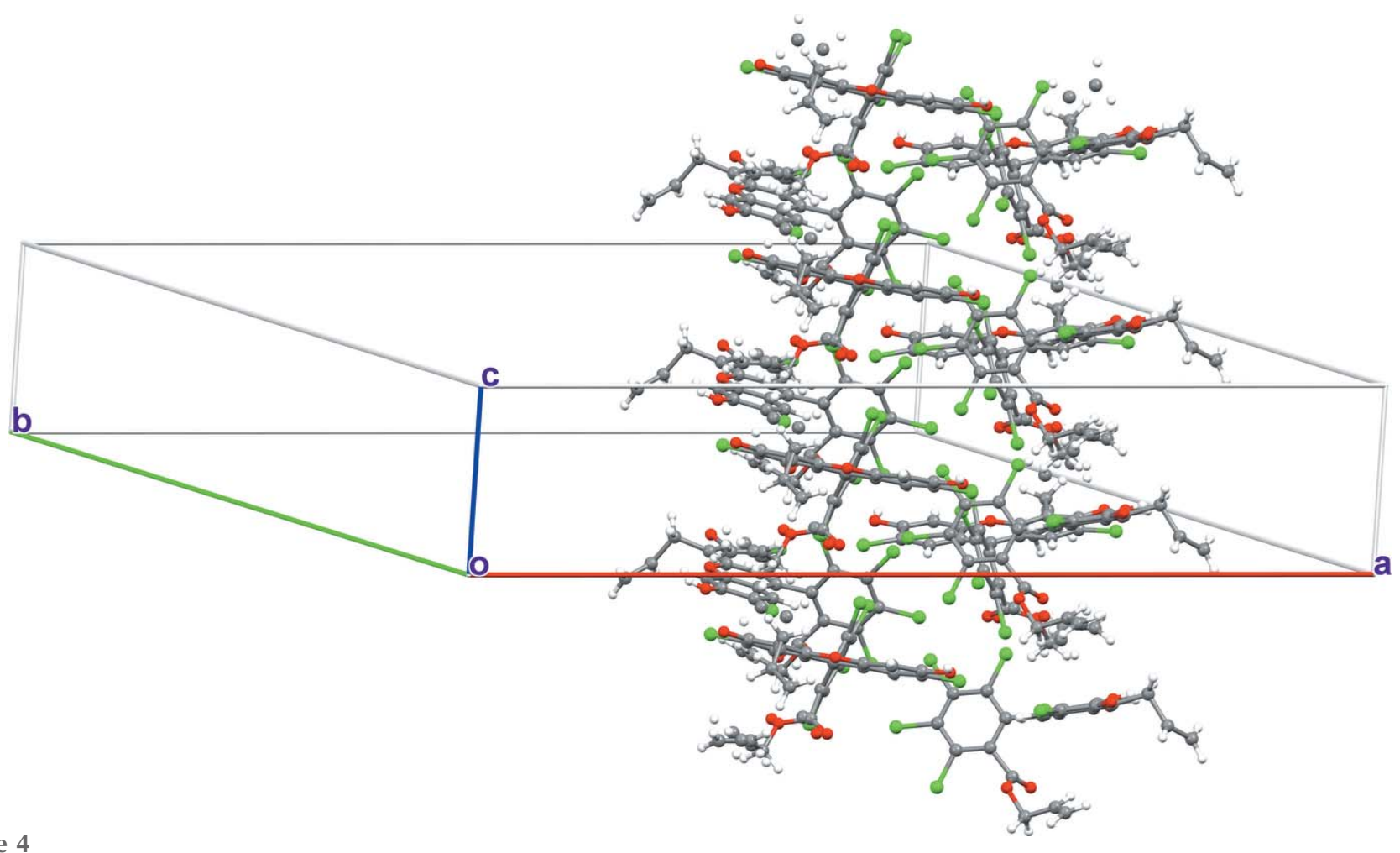

Figure 4

Three-dimensional packing diagram of the title compound.

$4,5,6,7,2^{\prime}, 7^{\prime}$-Hexachlorofluorescein diallyl ether ester (500 mg) in diphenyl ether $(5 \mathrm{ml})$ was heated in a sealed tube at $443 \mathrm{~K}$ under $\mathrm{N}_{2}$ overnight. The homogeneous mixture was then cooled to room temperature, transferred to a scintillation vial, and diluted with $\mathrm{CHCl}_{3}(5 \mathrm{ml})$. Red prismatic crystals of the title compound formed slowly from this mixture at room temperature within three months, yield: $52 \%$. This crystalline material contained 0.3 equiv. of diphenyl ether and $c a 0.1$ equiv of $\mathrm{CHCl}_{3}$, as determined by ${ }^{1} \mathrm{H}$ NMR integration. Note that the quantity of $\mathrm{CHCl}_{3}$ could be underestimated because of the overly long $T_{1}$ relaxation time of the $\mathrm{H}-\mathrm{CCl}_{3}$ proton. The volatile nature of $\mathrm{CHCl}_{3}$ and the loss in the sampledissolving process could also contribute to underestimation.

Data for the title compound: ${ }^{1} \mathrm{H}$ NMR (500 MHz, $\left.\mathrm{CD}_{3} \mathrm{OD}\right)$ : $\delta 7.25(s, 1 \mathrm{H}), 7.20(s, 1 \mathrm{H}), 7.03($ br s, $1 \mathrm{H}), 5.96(d d t, J=16.9$, $10.2,6.5 \mathrm{~Hz}, 1 \mathrm{H}), 5.32(d d t, J=17.0,10.4,6.5 \mathrm{~Hz}, 1 \mathrm{H}), 5.17(d q$, $J=17.1,1.7 \mathrm{~Hz}, 1 \mathrm{H}), 5.06-4.95(m, 3 \mathrm{H}), 4.45-4.41(m, 2 \mathrm{H})$, $3.58(d t, J=6.4,1.3 \mathrm{~Hz}, 2 \mathrm{H})$.

Data for diphenyl ether: ${ }^{1} \mathrm{H}$ NMR (500 MHz, $\left.\mathrm{CD}_{3} \mathrm{OD}\right): \delta$ 7.36-7.32 $(m, 4 \mathrm{H}), 7.10(t t, J=7.5,1.1 \mathrm{~Hz}, 2 \mathrm{H}), 6.98-6.96(m$, $4 \mathrm{H})$.

Data for $\mathrm{CHCl}_{3}:{ }^{1} \mathrm{H}$ NMR $\left(500 \mathrm{MHz}, \mathrm{CD}_{3} \mathrm{OD}\right): \delta 7.90(s$, $1 \mathrm{H})$.

HRMS (ESI-TOF, positive ion, $m / z)$ : Calc. $618.9022([\mathrm{M}+$ $\mathrm{H}]^{+}$), found 618.9015 .

\section{Refinement}

Crystal data, data collection and structural refinement details are summarized in Table 2. Carbon-bound $\mathrm{H}$ atoms were placed in calculated positions $(\mathrm{C}-\mathrm{H}=0.95-0.98 \AA)$ and were included in the refinement in the riding-model approximation, with $U_{\text {iso }}(\mathrm{H})$ set to $1.2-1.5 U_{\text {eq }}(\mathrm{C})$. The $\mathrm{H}$ atom of the hydroxyl group was found in a difference-Fourier map and freely refined $[\mathrm{O}-\mathrm{H}=0.74$ (4) $\AA$ ] . Most atoms except those of the allyl groups were refined anisotropically. Both allyl groups were found to be disordered and each disorder was individually modeled with the application of appropriate geometric (SADI) restraints or thermal parameters (EADP) constraints. The disorder was modelled over two positions (refined occupancies of 0.5:0.5 and 0.55:0.45). Similar distance soft restraints were used for the allyl groups. Hydrogen atoms were included in idealized positions for structure-factor calculations.

The crystal contained many disordered solvent molecules located in several solvent-accessible voids. ${ }^{1} \mathrm{H}$ NMR analysis of the crystalline material in MeOD revealed that both $\mathrm{Ph}_{2} \mathrm{O}$ and $\mathrm{CHCl}_{3}$ are present. The amount of $\mathrm{Ph}_{2} \mathrm{O}$ is quantified to be 0.3 equiv. using the integrals for multiplets at $\delta 7.37-7.32$ $(4 \mathrm{H}), 7.12-7.07(2 \mathrm{H})$, and $6.98-6.96(4 \mathrm{H})$. The amount of $\mathrm{CHCl}_{3}$ is found to be approximately 0.1 equiv. using the integral for the singlet at $\delta 7.90$. The amount of the $\mathrm{CHCl}_{3}$ is most probably underestimated owing to a very long T1 relaxation time of the $\mathrm{HCCl}_{3}$ proton and its loss in the sample during the dissolving process and crystals transfer. These results guided the disorder modeling of the allyl group pointing into the void as $0.5: 0.5$. The allyl group inside the void is poorly defined and could not be freely refined. Attempts to model the disordered solvent area were not successful, and the diffuse contribution to scattering was treated by application of 
Table 2

Experimental details.

\begin{tabular}{|c|c|}
\hline \multicolumn{2}{|l|}{ Crystal data } \\
\hline Chemical formula & $\mathrm{C}_{26} \mathrm{H}_{14} \mathrm{Cl}_{6} \mathrm{O}_{5}$ \\
\hline$M_{\mathrm{r}}$ & 619.07 \\
\hline Crystal system, space group & Tetragonal, $I 4_{1} / a$ \\
\hline Temperature $(\mathrm{K})$ & 100 \\
\hline$a, c(\AA)$ & $41.432(2), 7.7844(6)$ \\
\hline$V\left(\AA^{3}\right)$ & $13363.0(18)$ \\
\hline$Z$ & 16 \\
\hline Radiation type & Mo $K \alpha$ \\
\hline$\mu\left(\mathrm{mm}^{-1}\right)$ & 0.54 \\
\hline Crystal size (mm) & $0.42 \times 0.32 \times 0.18$ \\
\hline \multicolumn{2}{|l|}{ Data collection } \\
\hline Diffractometer & Bruker D8 Venture \\
\hline Absorption correction & $\begin{array}{l}\text { Multi-scan ( } S A D A B S ; \text { Bruker, } \\
\text { 2015) }\end{array}$ \\
\hline$T_{\min }, T_{\max }$ & $0.803,0.940$ \\
\hline $\begin{array}{l}\text { No. of measured, independent and } \\
\text { observed }[I>2 \sigma(I)] \text { reflections }\end{array}$ & $105222,7959,6656$ \\
\hline$R_{\text {int }}$ & 0.043 \\
\hline$(\sin \theta / \lambda)_{\max }\left(\AA^{-1}\right)$ & 0.659 \\
\hline \multicolumn{2}{|l|}{ Refinement } \\
\hline$R\left[F^{2}>2 \sigma\left(F^{2}\right)\right], w R\left(F^{2}\right), S$ & $0.055,0.156,1.05$ \\
\hline No. of reflections & 7959 \\
\hline No. of parameters & 327 \\
\hline No. of restraints & 13 \\
\hline H-atom treatment & $\begin{array}{l}\mathrm{H} \text { atoms treated by a mixture of } \\
\text { independent and constrained } \\
\text { refinement }\end{array}$ \\
\hline$\Delta \rho_{\max }, \Delta \rho_{\min }\left(\mathrm{e} \AA^{-3}\right)$ & $1.08,-0.69$ \\
\hline
\end{tabular}

Computer programs: APEX3 and SAINT (Bruker, 2015), SHELXT (Sheldrick, 2015a), SHELXL2017 (Sheldrick, 2015b) and OLEX2 (Dolomanov et al., 2009).

the SQUEEZE routine (Spek, 2015) as implemented in PLATON (Spek, 2009) using the fab file construct: the SQUEEZE algorithm located four voids, centered at $(0,0.250$, $0.625),(0,0.750,0.375),(0,0.250,0.875)$ and $(0,0.750,0.125)$ with a volume of $860 \AA^{3}$ and an electron count of 186 or approximately 47 electrons per molecule of fluorescein. From the ${ }^{1} \mathrm{H}$ NMR data, 0.3 equiv. of $\mathrm{Ph}_{2} \mathrm{O}$ and 0.2 equiv. of $\mathrm{CHCl}_{3}$ account for 39 electrons.

\section{Funding information}

The authors thank DTRA (Grant No. HDTRA1-10-0091), AFOSR (Grant No. FA9550-14-1-0367), DARPA QuBE (Grant No. N66001-10-1-4060), NSF MRSEC Program
(Grant No. DMR 14-20709), Vannevar Bush Fellowship (ONR N00014-16-1-2513), the Alfred P. Sloan Foundation, and the Camille and Henry Dreyfus Foundation for support.

\section{References}

Abrahams, B. F., McCormick, L. J. \& Robson, R. (2009). J. Mol. Struct. 920, 466-471.

Bruker (2015). SAINT, APEX3 and SADABS. Bruker AXS Inc., Madison, Wisconsin, USA.

Christianson, A. M. \& Gabbaï, F. P. (2016). Inorg. Chem. 55, 58285835.

Cody, V. (1987). Acta Cryst. C43, 705-707.

Dolomanov, O. V., Bourhis, L. J., Gildea, R. J., Howard, J. A. K. \& Puschmann, H. (2009). J. Appl. Cryst. 42, 339-341.

Dufresne, S., Pérez Guarìn, S. A. \& Skene, W. G. (2007). Acta Cryst. C63, o350-o352.

Giepmans, B. N. G., Adams, S. R., Ellisman, M. H. \& Tsien, R. Y. (2006). Science, 312, 217-224.

Groom, C. R., Bruno, I. J., Lightfoot, M. P. \& Ward, S. C. (2016). Acta Cryst. B72, 171-179.

Harrison, W. T. A., Ramadevi, P., Seethalakshmi, P. G. \& Kumaresan, S. (2007). Acta Cryst. E63, o3911.

Hou, F., Cheng, J., Xi, P., Chen, F., Huang, L., Xie, G., Shi, Y., Liu, H., Bai, D. \& Zeng, Z. (2012). Dalton Trans. 41, 5799-5804.

Li, X., Traganos, F., Melamed, M. R. \& Darzynkiewicz, Z. (1995). Cytometry, 20, 172-180.

Pepe, G., Cole, J. M., Waddell, P. G. \& Griffiths, J. R. D. (2016). Mol. Syst. Des. Eng. 1, 402-415.

Quint, V., Morlet-Savary, F., Lohier, J.-F., Lalevée, J., Gaumont, A.-C. \& Lakhdar, S. (2016). J. Am. Chem. Soc. 138, 7436-7441.

Sezukuri, K., Suzuki, M., Hayashi, H., Kuzuhara, D., Aratani, N. \& Yamada, H. (2016). Chem. Commun. 52, 4872-4875.

Sheldrick, G. M. (2015a). Acta Cryst. A71, 3-8.

Sheldrick, G. M. (2015b). Acta Cryst. C71, 3-8.

Spek, A. L. (2009). Acta Cryst. D65, 148-155.

Spek, A. L. (2015). Acta Cryst. C71, 9-18.

Swamy, K. M. K., Lee, Y. J., Lee, H. N., Chun, J., Kim, Y., Kim, S.-J. \& Yoon, J. (2006). J. Org. Chem. 71, 8626-8628.

Tremayne, M., Kariuki, B. M. \& Harris, K. D. M. (1997). Angew. Chem. Int. Ed. Engl. 36, 770-772.

Wang, L., Griffin, G. B., Zhang, A., Zhai, F., Williams, N. E., Jordan, R. F. \& Engel, G. S. (2017). Nat. Chem. 9, 219-225.

Wang, W., Rusin, O., Xu, X., Kim, K. K., Escobedo, J. O., Fakayode, S. O., Fletcher, K. A., Lowry, M., Schowalter, C. M., Lawrence, C. M., Fronczek, F. R., Warner, I. M. \& Strongin, R. M. (2005). J. Am. Chem. Soc. 127, 15949-15958.

Willner, I., Eichen, Y., Rabinovitz, M., Hoffman, R. \& Cohen, S. (1992). J. Am. Chem. Soc. 114, 637-644. 


\section{supporting information}

Acta Cryst. (2018). E74, 83-87 [https://doi.org/10.1107/S2056989017018163]

\section{Crystal structure of $4^{\prime}$-allyl-4,5,6,7,2',7'-hexachlorofluorescein allyl ester}

\section{unknown solvate}

\section{Lili Wang, Alexander S. Filatov and Gregory S. Engel}

Computing details

Data collection: APEX3 (Bruker, 2015); cell refinement: SAINT (Bruker, 2015); data reduction: SAINT (Bruker, 2015); program(s) used to solve structure: SHELXT (Sheldrick, 2015a); program(s) used to refine structure: SHELXL2017 (Sheldrick, 2015b); molecular graphics: OLEX2 (Dolomanov et al., 2009); software used to prepare material for publication: OLEX2 (Dolomanov et al., 2009).

Prop-2-en-1-yl 2,3,4,5-tetrachloro-6-[2,7-dichloro-6-hydroxy-3-oxo-4-(prop-2-en-1-yl)-3H-xanthen-9yl]benzoate

Crystal data

$\mathrm{C}_{26} \mathrm{H}_{14} \mathrm{Cl}_{6} \mathrm{O}_{5}$

$M_{r}=619.07$

Tetragonal, $I 4_{1} / a$

$a=41.432(2) \AA$

$c=7.7844(6) \AA$

$V=13363.0(18) \AA^{3}$

$Z=16$

$F(000)=4992$

\section{Data collection}

Bruker D8 Venture diffractometer

Detector resolution: 10.4167 pixels $\mathrm{mm}^{-1}$ $\omega$ and phi scans

Absorption correction: multi-scan

(SADABS; Bruker, 2015)

$T_{\min }=0.803, T_{\max }=0.940$

105222 measured reflections

\section{Refinement}

Refinement on $F^{2}$

Least-squares matrix: full

$R\left[F^{2}>2 \sigma\left(F^{2}\right)\right]=0.055$

$w R\left(F^{2}\right)=0.156$

$S=1.05$

7959 reflections

327 parameters

13 restraints

Primary atom site location: dual
$D_{\mathrm{x}}=1.231 \mathrm{Mg} \mathrm{m}^{-3}$

Mo $K \alpha$ radiation, $\lambda=0.71073 \AA$

Cell parameters from 9585 reflections

$\theta=2.2-27.9^{\circ}$

$\mu=0.54 \mathrm{~mm}^{-1}$

$T=100 \mathrm{~K}$

Prism, red

$0.42 \times 0.32 \times 0.18 \mathrm{~mm}$

7959 independent reflections

6656 reflections with $I>2 \sigma(I)$

$R_{\text {int }}=0.043$

$\theta_{\max }=27.9^{\circ}, \theta_{\min }=2.2^{\circ}$

$h=-54 \rightarrow 54$

$k=-54 \rightarrow 54$

$l=-9 \rightarrow 10$

Secondary atom site location: difference Fourier map

Hydrogen site location: mixed

$\mathrm{H}$ atoms treated by a mixture of independent and constrained refinement

$w=1 /\left[\sigma^{2}\left(F_{\mathrm{o}}^{2}\right)+(0.0825 P)^{2}+46.1798 P\right]$

where $P=\left(F_{\mathrm{o}}{ }^{2}+2 F_{\mathrm{c}}{ }^{2}\right) / 3$

$(\Delta / \sigma)_{\max }=0.001$

$\Delta \rho_{\max }=1.08 \mathrm{e} \AA^{-3}$

$\Delta \rho_{\min }=-0.69$ e $\AA^{-3}$ 


\section{Special details}

Geometry. All esds (except the esd in the dihedral angle between two 1.s. planes) are estimated using the full covariance matrix. The cell esds are taken into account individually in the estimation of esds in distances, angles and torsion angles; correlations between esds in cell parameters are only used when they are defined by crystal symmetry. An approximate (isotropic) treatment of cell esds is used for estimating esds involving l.s. planes.

Fractional atomic coordinates and isotropic or equivalent isotropic displacement parameters $\left(\AA^{2}\right)$

\begin{tabular}{|c|c|c|c|c|c|}
\hline & $x$ & $y$ & $z$ & $U_{\text {iso }} * / U_{\text {eq }}$ & Occ. $(<1)$ \\
\hline $\mathrm{Cl1}$ & $0.39543(2)$ & $0.48283(2)$ & $0.58350(11)$ & $0.03112(17)$ & \\
\hline $\mathrm{Cl} 2$ & $0.27839(2)$ & $0.26500(2)$ & $0.42276(14)$ & $0.0454(2)$ & \\
\hline $\mathrm{Cl} 3$ & $0.31144(2)$ & $0.41091(2)$ & $0.23233(8)$ & $0.02342(15)$ & \\
\hline $\mathrm{Cl} 4$ & $0.24970(2)$ & $0.45200(2)$ & $0.29555(8)$ & $0.02253(15)$ & \\
\hline $\mathrm{Cl} 5$ & $0.21385(2)$ & $0.44703(2)$ & $0.64520(9)$ & $0.02840(16)$ & \\
\hline $\mathrm{Cl} 6$ & $0.23918(2)$ & $0.40045(2)$ & $0.92801(9)$ & $0.03081(17)$ & \\
\hline $\mathrm{O} 2$ & $0.45019(5)$ & $0.44372(5)$ & $0.4967(4)$ & $0.0402(6)$ & \\
\hline $\mathrm{H} 2$ & $0.4637(10)$ & $0.4339(10)$ & $0.463(5)$ & $0.045(11)^{*}$ & \\
\hline $\mathrm{O} 3$ & $0.39550(4)$ & $0.34387(4)$ & 0.4569 (3) & $0.0305(5)$ & \\
\hline $\mathrm{O} 4$ & $0.34551(5)$ & $0.24470(5)$ & $0.3600(4)$ & $0.0467(7)$ & \\
\hline $\mathrm{C} 1$ & $0.42382(6)$ & $0.42571(6)$ & $0.5078(4)$ & $0.0286(6)$ & \\
\hline $\mathrm{C} 2$ & $0.39447(6)$ & $0.44152(6)$ & $0.5503(4)$ & $0.0247(5)$ & \\
\hline $\mathrm{C} 3$ & $0.36604(6)$ & $0.42505(6)$ & $0.5618(3)$ & $0.0212(5)$ & \\
\hline $\mathrm{H} 3$ & 0.346796 & 0.436173 & 0.591771 & $0.025^{*}$ & \\
\hline $\mathrm{C} 4$ & $0.36511(6)$ & $0.39153(6)$ & $0.5293(3)$ & $0.0189(5)$ & \\
\hline $\mathrm{C} 5$ & $0.39438(6)$ & $0.37617(6)$ & $0.4901(4)$ & $0.0242(5)$ & \\
\hline C6 & $0.42330(6)$ & $0.39261(7)$ & 0.4794 (4) & $0.0313(6)$ & \\
\hline H6 & 0.442660 & 0.381395 & 0.452782 & $0.038^{*}$ & \\
\hline $\mathrm{C} 7$ & $0.34334(7)$ & $0.27382(6)$ & $0.4012(5)$ & $0.0320(7)$ & \\
\hline $\mathrm{C} 8$ & $0.37143(7)$ & $0.29388(6)$ & $0.4114(5)$ & $0.0348(7)$ & \\
\hline $\mathrm{C} 9$ & $0.36809(6)$ & $0.32553(6)$ & 0.4539 (4) & $0.0244(5)$ & \\
\hline $\mathrm{C} 10$ & $0.33741(5)$ & $0.34042(5)$ & 0.4904 (3) & $0.0179(5)$ & \\
\hline C11 & $0.30949(6)$ & $0.32027(6)$ & $0.4812(3)$ & $0.0211(5)$ & \\
\hline H11 & 0.288808 & 0.329055 & 0.505540 & $0.025^{*}$ & \\
\hline $\mathrm{C} 12$ & $0.31228(6)$ & $0.28889(6)$ & $0.4383(4)$ & $0.0262(6)$ & \\
\hline $\mathrm{C} 13$ & $0.33624(5)$ & $0.37283(6)$ & $0.5269(3)$ & 0.0169 (4) & \\
\hline C14 & $0.30471(5)$ & $0.38966(5)$ & $0.5564(3)$ & 0.0158 (4) & \\
\hline $\mathrm{C} 15$ & $0.29226(5)$ & $0.40932(5)$ & $0.4272(3)$ & $0.0162(4)$ & \\
\hline $\mathrm{C} 16$ & $0.26413(5)$ & $0.42701(5)$ & $0.4541(3)$ & 0.0167 (4) & \\
\hline $\mathrm{C} 17$ & $0.24778(5)$ & $0.42433(6)$ & 0.6088 (3) & $0.0187(5)$ & \\
\hline $\mathrm{C} 18$ & $0.25919(6)$ & $0.40341(6)$ & $0.7354(3)$ & $0.0198(5)$ & \\
\hline C19 & $0.28768(6)$ & $0.38606(6)$ & 0.7089 & $0.0192(5)$ & \\
\hline $\mathrm{O} 5 \mathrm{~A}$ & $0.32942(12)$ & $0.37441(15)$ & $0.9111(6)$ & $0.0277(12)^{*}$ & $0.515(11)$ \\
\hline O6A & $0.28648(16)$ & $0.34190(13)$ & $0.8881(7)$ & $0.0337(14)^{*}$ & $0.515(11)$ \\
\hline $\mathrm{C} 20 \mathrm{~A}$ & $0.30476(16)$ & $0.36766(16)$ & $0.8496(8)$ & $0.0165(16)^{*}$ & $0.515(11)$ \\
\hline $\mathrm{C} 21 \mathrm{~A}$ & $0.2973(2)$ & $0.32063(19)$ & $1.0245(10)$ & $0.0398(17)^{*}$ & $0.515(11)$ \\
\hline $\mathrm{H} 21 \mathrm{~A}$ & 0.309106 & 0.333258 & 1.112086 & $0.048^{*}$ & $0.515(11)$ \\
\hline $\mathrm{H} 21 \mathrm{~B}$ & 0.278333 & 0.310548 & 1.080631 & $0.048^{*}$ & $0.515(11)$ \\
\hline $\mathrm{C} 22 \mathrm{~A}$ & $0.3178(2)$ & $0.29612(19)$ & $0.9564(11)$ & $0.058(2)^{*}$ & $0.515(11)$ \\
\hline
\end{tabular}




\begin{tabular}{|c|c|c|c|c|c|}
\hline $\mathrm{H} 22 \mathrm{~A}$ & 0.337240 & 0.303079 & 0.903352 & $0.069 *$ & $0.515(11)$ \\
\hline $\mathrm{C} 23 \mathrm{~A}$ & 0.3123 & $0.2639(2)$ & $0.9600(14)$ & $0.077(3)^{*}$ & $0.515(11)$ \\
\hline $\mathrm{H} 23 \mathrm{~A}$ & 0.293156 & 0.255742 & 1.011623 & $0.092 *$ & $0.515(11)$ \\
\hline $\mathrm{H} 23 \mathrm{~B}$ & 0.327502 & 0.249450 & 0.910923 & $0.092 *$ & $0.515(11)$ \\
\hline O5B & $0.32477(13)$ & $0.36512(17)$ & $0.9121(7)$ & $0.0307(13)^{*}$ & $0.485(11)$ \\
\hline O6B & $0.27778(16)$ & $0.33861(12)$ & $0.8719(7)$ & $0.0290(13)^{*}$ & $0.485(11)$ \\
\hline $\mathrm{C} 20 \mathrm{~B}$ & $0.29915(17)$ & $0.36200(18)$ & $0.8455(8)$ & $0.0162(17)^{*}$ & $0.485(11)$ \\
\hline $\mathrm{C} 21 \mathrm{~B}$ & $0.2864(2)$ & $0.3138(2)$ & $1.0020(11)$ & $0.046(2)^{*}$ & $0.485(11)$ \\
\hline $\mathrm{H} 21 \mathrm{C}$ & 0.302368 & 0.323054 & 1.082861 & $0.055^{*}$ & $0.485(11)$ \\
\hline H21D & 0.266885 & 0.308056 & 1.068539 & $0.055^{*}$ & $0.485(11)$ \\
\hline $\mathrm{C} 22 \mathrm{~B}$ & $0.2994(4)$ & $0.2857(4)$ & $0.928(2)$ & $0.111(5)^{*}$ & $0.485(11)$ \\
\hline $\mathrm{H} 22 \mathrm{~B}$ & 0.285565 & 0.271263 & 0.867772 & $0.134 *$ & $0.485(11)$ \\
\hline $\mathrm{C} 23 \mathrm{~B}$ & $0.3325(4)$ & $0.2785(5)$ & $0.942(3)$ & $0.142(7)^{*}$ & $0.485(11)$ \\
\hline $\mathrm{H} 23 \mathrm{C}$ & 0.346502 & 0.292773 & 1.001980 & $0.170^{*}$ & $0.485(11)$ \\
\hline $\mathrm{H} 23 \mathrm{D}$ & 0.340941 & 0.259395 & 0.891333 & $0.170^{*}$ & $0.485(11)$ \\
\hline C24 & $0.40398(15)$ & $0.28020(16)$ & $0.3449(9)$ & $0.0320(9)^{*}$ & 0.5 \\
\hline $\mathrm{H} 24 \mathrm{~A}$ & 0.400391 & 0.264945 & 0.248897 & $0.038^{*}$ & 0.5 \\
\hline $\mathrm{H} 24 \mathrm{~B}$ & 0.418248 & 0.297825 & 0.304871 & $0.038^{*}$ & 0.5 \\
\hline $\mathrm{C} 25$ & $0.4188(4)$ & $0.2628(3)$ & $0.4996(15)$ & $0.104(3)^{*}$ & 0.5 \\
\hline $\mathrm{H} 25$ & 0.405761 & 0.246742 & 0.551741 & $0.124^{*}$ & 0.5 \\
\hline $\mathrm{C} 26$ & $0.4489(5)$ & $0.2677(7)$ & $0.573(4)$ & $0.253(10)^{*}$ & 0.5 \\
\hline $\mathrm{H} 26 \mathrm{~A}$ & 0.463067 & 0.283467 & 0.526681 & $0.303^{*}$ & 0.5 \\
\hline $\mathrm{H} 26 \mathrm{~B}$ & 0.455306 & 0.255248 & 0.669565 & $0.303^{*}$ & 0.5 \\
\hline $\mathrm{C} 24 \mathrm{~A}$ & $0.40402(15)$ & $0.27709(16)$ & $0.4069(9)$ & $0.0320(9)^{*}$ & 0.5 \\
\hline $\mathrm{H} 24 \mathrm{C}$ & 0.419940 & 0.288767 & 0.478513 & $0.038 *$ & 0.5 \\
\hline $\mathrm{H} 24 \mathrm{D}$ & 0.402203 & 0.254619 & 0.448981 & $0.038 *$ & 0.5 \\
\hline $\mathrm{C} 25 \mathrm{~A}$ & $0.4134(3)$ & $0.2777(4)$ & $0.2274(16)$ & $0.104(3)^{*}$ & 0.5 \\
\hline $\mathrm{H} 25 \mathrm{~A}$ & 0.414367 & 0.296477 & 0.157479 & $0.124^{*}$ & 0.5 \\
\hline $\mathrm{C} 26 \mathrm{~A}$ & $0.4210(7)$ & $0.2454(6)$ & $0.171(4)$ & $0.253(10)^{*}$ & 0.5 \\
\hline $\mathrm{H} 26 \mathrm{C}$ & 0.419396 & 0.227889 & 0.249686 & $0.303 *$ & 0.5 \\
\hline $\mathrm{H} 26 \mathrm{D}$ & 0.427605 & 0.241649 & 0.056278 & $0.303^{*}$ & 0.5 \\
\hline
\end{tabular}

Atomic displacement parameters $\left(\AA^{2}\right)$

\begin{tabular}{lllllll}
\hline & $U^{11}$ & $U^{22}$ & $U^{33}$ & $U^{12}$ & $U^{13}$ & $U^{23}$ \\
\hline $\mathrm{C} 11$ & $0.0197(3)$ & $0.0175(3)$ & $0.0562(5)$ & $-0.0022(2)$ & $0.0040(3)$ & $-0.0126(3)$ \\
$\mathrm{C} 12$ & $0.0226(3)$ & $0.0189(3)$ & $0.0947(7)$ & $-0.0046(2)$ & $-0.0035(4)$ & $-0.0100(4)$ \\
$\mathrm{Cl} 3$ & $0.0204(3)$ & $0.0253(3)$ & $0.0245(3)$ & $0.0027(2)$ & $0.0056(2)$ & $0.0038(2)$ \\
$\mathrm{C} 14$ & $0.0212(3)$ & $0.0206(3)$ & $0.0258(3)$ & $0.0057(2)$ & $-0.0041(2)$ & $0.0019(2)$ \\
$\mathrm{C} 15$ & $0.0196(3)$ & $0.0342(3)$ & $0.0314(3)$ & $0.0135(2)$ & $0.0019(2)$ & $-0.0024(3)$ \\
$\mathrm{C} 16$ & $0.0300(3)$ & $0.0393(4)$ & $0.0231(3)$ & $0.0109(3)$ & $0.0075(3)$ & $0.0007(3)$ \\
$\mathrm{O} 2$ & $0.0132(9)$ & $0.0229(10)$ & $0.0844(19)$ & $-0.0038(8)$ & $0.0105(10)$ & $-0.0175(11)$ \\
$\mathrm{O} 3$ & $0.0138(8)$ & $0.0161(8)$ & $0.0617(14)$ & $0.0014(6)$ & $0.0016(9)$ & $-0.0128(9)$ \\
$\mathrm{O} 4$ & $0.0264(10)$ & $0.0162(9)$ & $0.098(2)$ & $0.0031(8)$ & $-0.0117(12)$ & $-0.0170(11)$ \\
$\mathrm{C} 1$ & $0.0125(11)$ & $0.0202(12)$ & $0.0529(18)$ & $-0.0005(9)$ & $0.0033(11)$ & $-0.0103(12)$ \\
$\mathrm{C} 2$ & $0.0184(12)$ & $0.0158(11)$ & $0.0400(15)$ & $-0.0010(9)$ & $0.0018(11)$ & $-0.0095(11)$ \\
$\mathrm{C} 3$ & $0.0127(10)$ & $0.0182(11)$ & $0.0328(14)$ & $0.0008(8)$ & $0.0015(10)$ & $-0.0071(10)$ \\
$\mathrm{C} 4$ & $0.0142(10)$ & $0.0156(11)$ & $0.0268(12)$ & $0.0018(8)$ & $0.0008(9)$ & $-0.0052(9)$
\end{tabular}




$\begin{array}{lllllll}\text { C5 } & 0.0168(11) & 0.0151(11) & 0.0407(15) & 0.0024(9) & -0.0002(11) & -0.0082(10) \\ \text { C6 } & 0.0139(11) & 0.0220(13) & 0.0579(19) & 0.0017(9) & 0.0042(12) & -0.0120(13) \\ \text { C7 } & 0.0226(13) & 0.0154(12) & 0.0580(19) & 0.0035(10) & -0.0084(13) & -0.0062(12) \\ \text { C8 } & 0.0206(13) & 0.0177(12) & 0.066(2) & 0.0050(10) & -0.0058(13) & -0.0106(13) \\ \text { C9 } & 0.0146(11) & 0.0165(11) & 0.0422(15) & 0.0014(9) & -0.0040(10) & -0.0049(11) \\ \text { C10 } & 0.0155(11) & 0.0139(10) & 0.0242(12) & 0.0023(8) & -0.0033(9) & -0.0014(9) \\ \text { C11 } & 0.0154(11) & 0.0173(11) & 0.0305(13) & 0.0012(9) & -0.0032(10) & -0.0004(10) \\ \text { C12 } & 0.0193(12) & 0.0146(11) & 0.0447(16) & -0.0013(9) & -0.0071(11) & -0.0033(11) \\ \text { C13 } & 0.0133(10) & 0.0161(11) & 0.0215(11) & 0.0013(8) & -0.0024(9) & -0.0009(9) \\ \text { C14 } & 0.0120(10) & 0.0116(10) & 0.0238(12) & -0.0012(8) & -0.0011(9) & -0.0043(9) \\ \text { C15 } & 0.0124(10) & 0.0131(10) & 0.0231(12) & -0.0020(8) & 0.0003(9) & -0.0018(9) \\ \text { C16 } & 0.0147(10) & 0.0122(10) & 0.0231(12) & 0.0003(8) & -0.0041(9) & -0.0014(9) \\ \text { C17 } & 0.0130(10) & 0.0173(11) & 0.0256(12) & 0.0015(8) & -0.0007(9) & -0.0053(9) \\ \text { C18 } & 0.0175(11) & 0.0221(12) & 0.0199(12) & 0.0016(9) & 0.0023(9) & -0.0052(9) \\ \text { C19 } & 0.0184(11) & 0.0170(11) & 0.0222(12) & 0.0034(9) & -0.0018(9) & -0.0033(9)\end{array}$

Geometric parameters $\left(\AA,{ }^{\circ}\right)$

\begin{tabular}{|c|c|c|c|}
\hline $\mathrm{C} 11-\mathrm{C} 2$ & $1.732(2)$ & $\mathrm{C} 19-\mathrm{C} 20 \mathrm{~A}$ & $1.511(6)$ \\
\hline $\mathrm{Cl} 2-\mathrm{C} 12$ & $1.722(3)$ & $\mathrm{C} 19-\mathrm{C} 20 \mathrm{~B}$ & $1.533(7)$ \\
\hline $\mathrm{C} 13-\mathrm{C} 15$ & $1.714(2)$ & $\mathrm{O} 5 \mathrm{~A}-\mathrm{C} 20 \mathrm{~A}$ & $1.162(8)$ \\
\hline $\mathrm{Cl} 4-\mathrm{C} 16$ & $1.718(2)$ & $\mathrm{O} 6 \mathrm{~A}-\mathrm{C} 20 \mathrm{~A}$ & $1.343(8)$ \\
\hline $\mathrm{C} 15-\mathrm{C} 17$ & $1.715(2)$ & O6A-C21A & $1.450(9)$ \\
\hline $\mathrm{Cl} 1-\mathrm{C} 18$ & $1.718(3)$ & $\mathrm{C} 21 \mathrm{~A}-\mathrm{H} 21 \mathrm{~A}$ & 0.9900 \\
\hline $\mathrm{O} 2-\mathrm{H} 2$ & $0.74(4)$ & $\mathrm{C} 21 \mathrm{~A}-\mathrm{H} 21 \mathrm{~B}$ & 0.9900 \\
\hline $\mathrm{O} 2-\mathrm{C} 1$ & $1.326(3)$ & $\mathrm{C} 21 \mathrm{~A}-\mathrm{C} 22 \mathrm{~A}$ & $1.427(10)$ \\
\hline $\mathrm{O} 3-\mathrm{C} 5$ & $1.364(3)$ & $\mathrm{C} 22 \mathrm{~A}-\mathrm{H} 22 \mathrm{~A}$ & 0.9500 \\
\hline $\mathrm{O} 3-\mathrm{C} 9$ & $1.367(3)$ & $\mathrm{C} 22 \mathrm{~A}-\mathrm{C} 23 \mathrm{~A}$ & $1.355(11)$ \\
\hline $\mathrm{O} 4-\mathrm{C} 7$ & $1.251(3)$ & $\mathrm{C} 23 \mathrm{~A}-\mathrm{H} 23 \mathrm{~A}$ & 0.9500 \\
\hline $\mathrm{C} 1-\mathrm{C} 2$ & $1.420(3)$ & $\mathrm{C} 23 \mathrm{~A}-\mathrm{H} 23 \mathrm{~B}$ & 0.9500 \\
\hline $\mathrm{C} 1-\mathrm{C} 6$ & $1.389(4)$ & $\mathrm{O} 5 \mathrm{~B}-\mathrm{C} 20 \mathrm{~B}$ & $1.188(8)$ \\
\hline $\mathrm{C} 2-\mathrm{C} 3$ & $1.364(3)$ & $\mathrm{O} 6 \mathrm{~B}-\mathrm{C} 20 \mathrm{~B}$ & $1.329(9)$ \\
\hline $\mathrm{C} 3-\mathrm{H} 3$ & 0.9500 & $\mathrm{O} 6 \mathrm{~B}-\mathrm{C} 21 \mathrm{~B}$ & $1.486(10)$ \\
\hline $\mathrm{C} 3-\mathrm{C} 4$ & $1.412(3)$ & $\mathrm{C} 21 \mathrm{~B}-\mathrm{H} 21 \mathrm{C}$ & 0.9900 \\
\hline $\mathrm{C} 4-\mathrm{C} 5$ & $1.403(3)$ & $\mathrm{C} 21 \mathrm{~B}-\mathrm{H} 21 \mathrm{D}$ & 0.9900 \\
\hline $\mathrm{C} 4-\mathrm{C} 13$ & $1.425(3)$ & $\mathrm{C} 21 \mathrm{~B}-\mathrm{C} 22 \mathrm{~B}$ & $1.407(18)$ \\
\hline $\mathrm{C} 5-\mathrm{C} 6$ & $1.381(4)$ & $\mathrm{C} 22 \mathrm{~B}-\mathrm{H} 22 \mathrm{~B}$ & 0.9500 \\
\hline $\mathrm{C} 6-\mathrm{H} 6$ & 0.9500 & $\mathrm{C} 22 \mathrm{~B}-\mathrm{C} 23 \mathrm{~B}$ & $1.411(15)$ \\
\hline $\mathrm{C} 7-\mathrm{C} 8$ & $1.432(4)$ & $\mathrm{C} 23 \mathrm{~B}-\mathrm{H} 23 \mathrm{C}$ & 0.9500 \\
\hline $\mathrm{C} 7-\mathrm{C} 12$ & $1.459(4)$ & $\mathrm{C} 23 \mathrm{~B}-\mathrm{H} 23 \mathrm{D}$ & 0.9500 \\
\hline $\mathrm{C} 8-\mathrm{C} 9$ & $1.359(4)$ & $\mathrm{C} 24-\mathrm{H} 24 \mathrm{~A}$ & 0.9900 \\
\hline $\mathrm{C} 8-\mathrm{C} 24$ & $1.552(7)$ & $\mathrm{C} 24-\mathrm{H} 24 \mathrm{~B}$ & 0.9900 \\
\hline $\mathrm{C} 8-\mathrm{C} 24 \mathrm{~A}$ & $1.519(6)$ & $\mathrm{C} 24-\mathrm{C} 25$ & $1.531(8)$ \\
\hline $\mathrm{C} 9-\mathrm{C} 10$ & $1.441(3)$ & $\mathrm{C} 25-\mathrm{H} 25$ & 0.9500 \\
\hline $\mathrm{C} 10-\mathrm{C} 11$ & $1.429(3)$ & $\mathrm{C} 25-\mathrm{C} 26$ & $1.385(17)$ \\
\hline $\mathrm{C} 10-\mathrm{C} 13$ & $1.373(3)$ & $\mathrm{C} 26-\mathrm{H} 26 \mathrm{~A}$ & 0.9500 \\
\hline $\mathrm{C} 11-\mathrm{H} 11$ & 0.9500 & $\mathrm{C} 26-\mathrm{H} 26 \mathrm{~B}$ & 0.9500 \\
\hline $\mathrm{C} 11-\mathrm{C} 12$ & $1.347(3)$ & $\mathrm{C} 24 \mathrm{~A}-\mathrm{H} 24 \mathrm{C}$ & 0.9900 \\
\hline
\end{tabular}


$\mathrm{C} 13-\mathrm{C} 14$
$\mathrm{C} 14-\mathrm{C} 15$
$\mathrm{C} 14-\mathrm{C} 19$
$\mathrm{C} 15-\mathrm{C} 16$
$\mathrm{C} 16-\mathrm{C} 17$
$\mathrm{C} 17-\mathrm{C} 18$
$\mathrm{C} 18-\mathrm{C} 19$

$\mathrm{C} 1-\mathrm{O} 2-\mathrm{H} 2$

$\mathrm{C} 5-\mathrm{O} 3-\mathrm{C} 9$

$\mathrm{O} 2-\mathrm{C} 1-\mathrm{C} 2$

$\mathrm{O} 2-\mathrm{C} 1-\mathrm{C} 6$

$\mathrm{C} 6-\mathrm{C} 1-\mathrm{C} 2$

$\mathrm{C} 1-\mathrm{C} 2-\mathrm{C} 1$

$\mathrm{C} 3-\mathrm{C} 2-\mathrm{C} 11$

$\mathrm{C} 3-\mathrm{C} 2-\mathrm{C} 1$

$\mathrm{C} 2-\mathrm{C} 3-\mathrm{H} 3$

$\mathrm{C} 2-\mathrm{C} 3-\mathrm{C} 4$

$\mathrm{C} 4-\mathrm{C} 3-\mathrm{H} 3$

$\mathrm{C} 3-\mathrm{C} 4-\mathrm{C} 13$

$\mathrm{C} 5-\mathrm{C} 4-\mathrm{C} 3$

$\mathrm{C} 5-\mathrm{C} 4-\mathrm{C} 13$

$\mathrm{O} 3-\mathrm{C} 5-\mathrm{C} 4$

$\mathrm{O} 3-\mathrm{C} 5-\mathrm{C} 6$

$\mathrm{C} 6-\mathrm{C} 5-\mathrm{C} 4$

$\mathrm{C} 1-\mathrm{C} 6-\mathrm{H} 6$

$\mathrm{C} 5-\mathrm{C} 6-\mathrm{C} 1$

$\mathrm{C} 5-\mathrm{C} 6-\mathrm{H} 6$

$\mathrm{O} 4-\mathrm{C} 7-\mathrm{C} 8$

$\mathrm{O} 4-\mathrm{C} 7-\mathrm{C} 12$

$\mathrm{C} 8-\mathrm{C} 7-\mathrm{C} 12$

$\mathrm{C} 7-\mathrm{C} 8-\mathrm{C} 24$

$\mathrm{C} 7-\mathrm{C} 8-\mathrm{C} 24 \mathrm{~A}$

$\mathrm{C} 9-\mathrm{C} 8-\mathrm{C} 7$

$\mathrm{C} 9-\mathrm{C} 8-\mathrm{C} 24$

$\mathrm{C} 9-\mathrm{C} 8-\mathrm{C} 24 \mathrm{~A}$

$\mathrm{O} 3-\mathrm{C} 9-\mathrm{C} 10$

$\mathrm{C} 8-\mathrm{C} 9-\mathrm{O} 3$

$\mathrm{C} 8-\mathrm{C} 9-\mathrm{C} 10$

$\mathrm{C} 11-\mathrm{C} 10-\mathrm{C} 9$

$\mathrm{C} 13-\mathrm{C} 10-\mathrm{C} 9$

$\mathrm{C} 13-\mathrm{C} 10-\mathrm{C} 11$

$\mathrm{C} 10-\mathrm{C} 11-\mathrm{H} 11$

$\mathrm{C} 12-\mathrm{C} 11-\mathrm{C} 10$

$\mathrm{C} 12-\mathrm{C} 11-\mathrm{H} 11$

$\mathrm{C} 7-\mathrm{C} 12-\mathrm{Cl} 2$

$\mathrm{C} 11-\mathrm{C} 12-\mathrm{Cl} 2$

$\mathrm{C} 11-\mathrm{C} 12-\mathrm{C} 7$
1.499 (3)

$1.393(3)$

$1.389(3)$

$1.392(3)$

1.387 (3)

1.395 (4)

1.397 (3)

110 (3)

121.41 (19)

$117.5(2)$

$123.9(2)$

$118.6(2)$

118.09 (19)

120.30 (19)

$121.6(2)$

119.9

120.2 (2)

119.9

$124.0(2)$

117.5 (2)

118.4 (2)

$121.0(2)$

116.3 (2)

$122.6(2)$

120.3

119.4 (2)

120.3

$121.0(3)$

121.8 (2)

$117.2(2)$

118.4 (3)

$117.1(3)$

119.4 (2)

$121.5(3)$

$122.5(3)$

119.4 (2)

117.1 (2)

123.4 (2)

117.0 (2)

119.4 (2)

$123.6(2)$

119.8

120.5 (2)

119.8

117.33 (19)

120.1 (2)

$122.5(2)$

$$
\begin{aligned}
& \mathrm{C} 24 \mathrm{~A}-\mathrm{H} 24 \mathrm{D} \\
& \mathrm{C} 24 \mathrm{~A}-\mathrm{C} 25 \mathrm{~A} \\
& \mathrm{C} 25 \mathrm{~A}-\mathrm{H} 25 \mathrm{~A} \\
& \mathrm{C} 25 \mathrm{~A}-\mathrm{C} 26 \mathrm{~A} \\
& \mathrm{C} 26 \mathrm{~A}-\mathrm{H} 26 \mathrm{C} \\
& \mathrm{C} 26 \mathrm{~A}-\mathrm{H} 26 \mathrm{D}
\end{aligned}
$$

C14-C19-C20B

$\mathrm{C} 18-\mathrm{C} 19-\mathrm{C} 20 \mathrm{~A}$

C18-C19-C20B

$\mathrm{C} 20 \mathrm{~A}-\mathrm{O} 6 \mathrm{~A}-\mathrm{C} 21 \mathrm{~A}$

$\mathrm{O} 5 \mathrm{~A}-\mathrm{C} 20 \mathrm{~A}-\mathrm{C} 19$

$\mathrm{O} 5 \mathrm{~A}-\mathrm{C} 20 \mathrm{~A}-\mathrm{O} 6 \mathrm{~A}$

O6A-C20A-C19

O6A-C21A-H21A

O6A-C21A-H21B

$\mathrm{H} 21 \mathrm{~A}-\mathrm{C} 21 \mathrm{~A}-\mathrm{H} 21 \mathrm{~B}$

$\mathrm{C} 22 \mathrm{~A}-\mathrm{C} 21 \mathrm{~A}-\mathrm{O} 6 \mathrm{~A}$

$\mathrm{C} 22 \mathrm{~A}-\mathrm{C} 21 \mathrm{~A}-\mathrm{H} 21 \mathrm{~A}$

$\mathrm{C} 22 \mathrm{~A}-\mathrm{C} 21 \mathrm{~A}-\mathrm{H} 21 \mathrm{~B}$

$\mathrm{C} 21 \mathrm{~A}-\mathrm{C} 22 \mathrm{~A}-\mathrm{H} 22 \mathrm{~A}$

$\mathrm{C} 23 \mathrm{~A}-\mathrm{C} 22 \mathrm{~A}-\mathrm{C} 21 \mathrm{~A}$

$\mathrm{C} 23 \mathrm{~A}-\mathrm{C} 22 \mathrm{~A}-\mathrm{H} 22 \mathrm{~A}$

$\mathrm{C} 22 \mathrm{~A}-\mathrm{C} 23 \mathrm{~A}-\mathrm{H} 23 \mathrm{~A}$

$\mathrm{C} 22 \mathrm{~A}-\mathrm{C} 23 \mathrm{~A}-\mathrm{H} 23 \mathrm{~B}$

$\mathrm{H} 23 \mathrm{~A}-\mathrm{C} 23 \mathrm{~A}-\mathrm{H} 23 \mathrm{~B}$

$\mathrm{C} 20 \mathrm{~B}-\mathrm{O} 6 \mathrm{~B}-\mathrm{C} 21 \mathrm{~B}$

$\mathrm{O} 5 \mathrm{~B}-\mathrm{C} 20 \mathrm{~B}-\mathrm{C} 19$

$\mathrm{O} 5 \mathrm{~B}-\mathrm{C} 20 \mathrm{~B}-\mathrm{O} 6 \mathrm{~B}$

$\mathrm{O} 6 \mathrm{~B}-\mathrm{C} 20 \mathrm{~B}-\mathrm{C} 19$

$\mathrm{O} 6 \mathrm{~B}-\mathrm{C} 21 \mathrm{~B}-\mathrm{H} 21 \mathrm{C}$

$\mathrm{O} 6 \mathrm{~B}-\mathrm{C} 21 \mathrm{~B}-\mathrm{H} 21 \mathrm{D}$

$\mathrm{H} 21 \mathrm{C}-\mathrm{C} 21 \mathrm{~B}-\mathrm{H} 21 \mathrm{D}$

$\mathrm{C} 22 \mathrm{~B}-\mathrm{C} 21 \mathrm{~B}-\mathrm{O} 6 \mathrm{~B}$

$\mathrm{C} 22 \mathrm{~B}-\mathrm{C} 21 \mathrm{~B}-\mathrm{H} 21 \mathrm{C}$

$\mathrm{C} 22 \mathrm{~B}-\mathrm{C} 21 \mathrm{~B}-\mathrm{H} 21 \mathrm{D}$

$\mathrm{C} 21 \mathrm{~B}-\mathrm{C} 22 \mathrm{~B}-\mathrm{H} 22 \mathrm{~B}$

$\mathrm{C} 21 \mathrm{~B}-\mathrm{C} 22 \mathrm{~B}-\mathrm{C} 23 \mathrm{~B}$

$\mathrm{C} 23 \mathrm{~B}-\mathrm{C} 22 \mathrm{~B}-\mathrm{H} 22 \mathrm{~B}$

$\mathrm{C} 22 \mathrm{~B}-\mathrm{C} 23 \mathrm{~B}-\mathrm{H} 23 \mathrm{C}$

$\mathrm{C} 22 \mathrm{~B}-\mathrm{C} 23 \mathrm{~B}-\mathrm{H} 23 \mathrm{D}$

$\mathrm{H} 23 \mathrm{C}-\mathrm{C} 23 \mathrm{~B}-\mathrm{H} 23 \mathrm{D}$

$\mathrm{C} 8-\mathrm{C} 24-\mathrm{H} 24 \mathrm{~A}$

$\mathrm{C} 8-\mathrm{C} 24-\mathrm{H} 24 \mathrm{~B}$

$\mathrm{H} 24 \mathrm{~A}-\mathrm{C} 24-\mathrm{H} 24 \mathrm{~B}$

$\mathrm{C} 25-\mathrm{C} 24-\mathrm{C} 8$

$\mathrm{C} 25-\mathrm{C} 24-\mathrm{H} 24 \mathrm{~A}$
0.9900

1.451 (12)

0.9500

1.442 (17)

0.9500

0.9500

120.3 (3)

$123.2(3)$

$119.6(3)$

118.2 (6)

126.1 (6)

126.5 (6)

107.4 (5)

109.6

109.6

108.1

110.1 (6)

109.6

109.6

116.8

126.3 (10)

116.8

120.0

120.0

120.0

116.7 (6)

$120.6(6)$

$127.4(6)$

112.0 (5)

109.0

109.0

107.8

112.7 (9)

109.0

109.0

119.5

121.0 (17)

119.5

120.0

120.0

120.0

110.8

110.8

108.8

105.0 (7)

110.8 


\begin{tabular}{|c|c|c|c|}
\hline $\mathrm{C} 4-\mathrm{C} 13-\mathrm{C} 14$ & $118.5(2)$ & $\mathrm{C} 25-\mathrm{C} 24-\mathrm{H} 24 \mathrm{~B}$ & 110.8 \\
\hline $\mathrm{C} 10-\mathrm{C} 13-\mathrm{C} 4$ & $120.3(2)$ & $\mathrm{C} 24-\mathrm{C} 25-\mathrm{H} 25$ & 115.9 \\
\hline $\mathrm{C} 10-\mathrm{C} 13-\mathrm{C} 14$ & $121.2(2)$ & $\mathrm{C} 26-\mathrm{C} 25-\mathrm{C} 24$ & $128.1(18)$ \\
\hline $\mathrm{C} 15-\mathrm{C} 14-\mathrm{C} 13$ & $119.0(2)$ & $\mathrm{C} 26-\mathrm{C} 25-\mathrm{H} 25$ & 115.9 \\
\hline $\mathrm{C} 19-\mathrm{C} 14-\mathrm{C} 13$ & $121.6(2)$ & $\mathrm{C} 25-\mathrm{C} 26-\mathrm{H} 26 \mathrm{~A}$ & 120.0 \\
\hline $\mathrm{C} 19-\mathrm{C} 14-\mathrm{C} 15$ & $119.4(2)$ & $\mathrm{C} 25-\mathrm{C} 26-\mathrm{H} 26 \mathrm{~B}$ & 120.0 \\
\hline $\mathrm{C} 14-\mathrm{C} 15-\mathrm{Cl} 3$ & $119.32(17)$ & $\mathrm{H} 26 \mathrm{~A}-\mathrm{C} 26-\mathrm{H} 26 \mathrm{~B}$ & 120.0 \\
\hline $\mathrm{C} 16-\mathrm{C} 15-\mathrm{Cl} 3$ & $120.06(19)$ & $\mathrm{C} 8-\mathrm{C} 24 \mathrm{~A}-\mathrm{H} 24 \mathrm{C}$ & 110.8 \\
\hline $\mathrm{C} 16-\mathrm{C} 15-\mathrm{C} 14$ & $120.6(2)$ & $\mathrm{C} 8-\mathrm{C} 24 \mathrm{~A}-\mathrm{H} 24 \mathrm{D}$ & 110.8 \\
\hline $\mathrm{C} 15-\mathrm{C} 16-\mathrm{Cl} 4$ & $120.03(19)$ & $\mathrm{H} 24 \mathrm{C}-\mathrm{C} 24 \mathrm{~A}-\mathrm{H} 24 \mathrm{D}$ & 108.9 \\
\hline $\mathrm{C} 17-\mathrm{C} 16-\mathrm{Cl} 4$ & $120.14(18)$ & $\mathrm{C} 25 \mathrm{~A}-\mathrm{C} 24 \mathrm{~A}-\mathrm{C} 8$ & $104.6(7)$ \\
\hline $\mathrm{C} 17-\mathrm{C} 16-\mathrm{C} 15$ & $119.8(2)$ & $\mathrm{C} 25 \mathrm{~A}-\mathrm{C} 24 \mathrm{~A}-\mathrm{H} 24 \mathrm{C}$ & 110.8 \\
\hline $\mathrm{C} 16-\mathrm{C} 17-\mathrm{Cl} 5$ & $120.01(19)$ & $\mathrm{C} 25 \mathrm{~A}-\mathrm{C} 24 \mathrm{~A}-\mathrm{H} 24 \mathrm{D}$ & 110.8 \\
\hline $\mathrm{C} 16-\mathrm{C} 17-\mathrm{C} 18$ & $119.8(2)$ & $\mathrm{C} 24 \mathrm{~A}-\mathrm{C} 25 \mathrm{~A}-\mathrm{H} 25 \mathrm{~A}$ & 125.3 \\
\hline $\mathrm{C} 18-\mathrm{C} 17-\mathrm{Cl} 5$ & $120.13(19)$ & $\mathrm{C} 26 \mathrm{~A}-\mathrm{C} 25 \mathrm{~A}-\mathrm{C} 24 \mathrm{~A}$ & $109.5(17)$ \\
\hline $\mathrm{C} 17-\mathrm{C} 18-\mathrm{Cl} 6$ & $119.82(18)$ & $\mathrm{C} 26 \mathrm{~A}-\mathrm{C} 25 \mathrm{~A}-\mathrm{H} 25 \mathrm{~A}$ & 125.3 \\
\hline $\mathrm{C} 17-\mathrm{C} 18-\mathrm{C} 19$ & $120.1(2)$ & $\mathrm{C} 25 \mathrm{~A}-\mathrm{C} 26 \mathrm{~A}-\mathrm{H} 26 \mathrm{C}$ & 120.0 \\
\hline $\mathrm{C} 19-\mathrm{C} 18-\mathrm{Cl} 6$ & $120.0(2)$ & $\mathrm{C} 25 \mathrm{~A}-\mathrm{C} 26 \mathrm{~A}-\mathrm{H} 26 \mathrm{D}$ & 120.0 \\
\hline $\mathrm{C} 14-\mathrm{C} 19-\mathrm{C} 18$ & $120.0(2)$ & $\mathrm{H} 26 \mathrm{C}-\mathrm{C} 26 \mathrm{~A}-\mathrm{H} 26 \mathrm{D}$ & 120.0 \\
\hline $\mathrm{C} 14-\mathrm{C} 19-\mathrm{C} 20 \mathrm{~A}$ & $115.8(3)$ & & \\
\hline $\mathrm{C} 11-\mathrm{C} 2-\mathrm{C} 3-\mathrm{C} 4$ & $178.0(2)$ & $\mathrm{C} 9-\mathrm{C} 10-\mathrm{C} 13-\mathrm{C} 4$ & $-0.3(4)$ \\
\hline $\mathrm{Cl} 3-\mathrm{C} 15-\mathrm{C} 16-\mathrm{Cl} 4$ & $-3.2(3)$ & $\mathrm{C} 9-\mathrm{C} 10-\mathrm{C} 13-\mathrm{C} 14$ & $176.7(2)$ \\
\hline $\mathrm{Cl} 3-\mathrm{C} 15-\mathrm{C} 16-\mathrm{C} 17$ & $176.60(17)$ & $\mathrm{C} 10-\mathrm{C} 11-\mathrm{C} 12-\mathrm{Cl} 2$ & $-178.5(2)$ \\
\hline $\mathrm{C} 14-\mathrm{C} 16-\mathrm{C} 17-\mathrm{Cl} 5$ & $-2.3(3)$ & $\mathrm{C} 10-\mathrm{C} 11-\mathrm{C} 12-\mathrm{C} 7$ & $0.6(4)$ \\
\hline $\mathrm{C} 14-\mathrm{C} 16-\mathrm{C} 17-\mathrm{C} 18$ & $178.46(18)$ & $\mathrm{C} 10-\mathrm{C} 13-\mathrm{C} 14-\mathrm{C} 15$ & $-104.3(3)$ \\
\hline $\mathrm{C} 15-\mathrm{C} 17-\mathrm{C} 18-\mathrm{Cl} 6$ & $0.0(3)$ & $\mathrm{C} 10-\mathrm{C} 13-\mathrm{C} 14-\mathrm{C} 19$ & $75.6(3)$ \\
\hline $\mathrm{C} 15-\mathrm{C} 17-\mathrm{C} 18-\mathrm{C} 19$ & $-176.96(19)$ & $\mathrm{C} 11-\mathrm{C} 10-\mathrm{C} 13-\mathrm{C} 4$ & $-178.3(2)$ \\
\hline $\mathrm{Cl} 6-\mathrm{C} 18-\mathrm{C} 19-\mathrm{C} 14$ & $-176.83(18)$ & $\mathrm{C} 11-\mathrm{C} 10-\mathrm{C} 13-\mathrm{C} 14$ & $-1.4(4)$ \\
\hline $\mathrm{Cl} 6-\mathrm{C} 18-\mathrm{C} 19-\mathrm{C} 20 \mathrm{~A}$ & $-8.2(5)$ & $\mathrm{C} 12-\mathrm{C} 7-\mathrm{C} 8-\mathrm{C} 9$ & $-0.2(5)$ \\
\hline $\mathrm{Cl} 6-\mathrm{C} 18-\mathrm{C} 19-\mathrm{C} 20 \mathrm{~B}$ & $5.8(5)$ & $\mathrm{C} 12-\mathrm{C} 7-\mathrm{C} 8-\mathrm{C} 24$ & $-170.4(4)$ \\
\hline $\mathrm{O} 2-\mathrm{C} 1-\mathrm{C} 2-\mathrm{Cl} 1$ & $0.1(4)$ & $\mathrm{C} 12-\mathrm{C} 7-\mathrm{C} 8-\mathrm{C} 24 \mathrm{~A}$ & $168.5(4)$ \\
\hline $\mathrm{O} 2-\mathrm{C} 1-\mathrm{C} 2-\mathrm{C} 3$ & $178.9(3)$ & $\mathrm{C} 13-\mathrm{C} 4-\mathrm{C} 5-\mathrm{O} 3$ & $-2.5(4)$ \\
\hline $\mathrm{O} 2-\mathrm{C} 1-\mathrm{C} 6-\mathrm{C} 5$ & $-178.4(3)$ & $\mathrm{C} 13-\mathrm{C} 4-\mathrm{C} 5-\mathrm{C} 6$ & $176.1(3)$ \\
\hline $\mathrm{O} 3-\mathrm{C} 5-\mathrm{C} 6-\mathrm{C} 1$ & $178.6(3)$ & $\mathrm{C} 13-\mathrm{C} 10-\mathrm{C} 11-\mathrm{C} 12$ & $177.5(3)$ \\
\hline $\mathrm{O} 3-\mathrm{C} 9-\mathrm{C} 10-\mathrm{C} 11$ & $178.5(2)$ & $\mathrm{C} 13-\mathrm{C} 14-\mathrm{C} 15-\mathrm{Cl} 3$ & $5.6(3)$ \\
\hline $\mathrm{O} 3-\mathrm{C} 9-\mathrm{C} 10-\mathrm{C} 13$ & $0.3(4)$ & $\mathrm{C} 13-\mathrm{C} 14-\mathrm{C} 15-\mathrm{C} 16$ & $-175.8(2)$ \\
\hline $\mathrm{O} 4-\mathrm{C} 7-\mathrm{C} 8-\mathrm{C} 9$ & $179.0(3)$ & $\mathrm{C} 13-\mathrm{C} 14-\mathrm{C} 19-\mathrm{C} 18$ & $176.7(2)$ \\
\hline $\mathrm{O} 4-\mathrm{C} 7-\mathrm{C} 8-\mathrm{C} 24$ & $8.8(6)$ & $\mathrm{C} 13-\mathrm{C} 14-\mathrm{C} 19-\mathrm{C} 20 \mathrm{~A}$ & $7.3(4)$ \\
\hline $\mathrm{O} 4-\mathrm{C} 7-\mathrm{C} 8-\mathrm{C} 24 \mathrm{~A}$ & $-12.3(6)$ & $\mathrm{C} 13-\mathrm{C} 14-\mathrm{C} 19-\mathrm{C} 20 \mathrm{~B}$ & $-6.0(5)$ \\
\hline $\mathrm{O} 4-\mathrm{C} 7-\mathrm{C} 12-\mathrm{Cl} 2$ & $-0.2(5)$ & $\mathrm{C} 14-\mathrm{C} 15-\mathrm{C} 16-\mathrm{Cl} 4$ & $178.20(17)$ \\
\hline $\mathrm{O} 4-\mathrm{C} 7-\mathrm{C} 12-\mathrm{C} 11$ & $-179.4(3)$ & $\mathrm{C} 14-\mathrm{C} 15-\mathrm{C} 16-\mathrm{C} 17$ & $-2.0(3)$ \\
\hline $\mathrm{C} 1-\mathrm{C} 2-\mathrm{C} 3-\mathrm{C} 4$ & $-0.7(4)$ & $\mathrm{C} 14-\mathrm{C} 19-\mathrm{C} 20 \mathrm{~A}-\mathrm{O} 5 \mathrm{~A}$ & $58.7(7)$ \\
\hline $\mathrm{C} 2-\mathrm{C} 1-\mathrm{C} 6-\mathrm{C} 5$ & $1.1(5)$ & $\mathrm{C} 14-\mathrm{C} 19-\mathrm{C} 20 \mathrm{~A}-\mathrm{O} 6 \mathrm{~A}$ & $-120.2(4)$ \\
\hline $\mathrm{C} 2-\mathrm{C} 3-\mathrm{C} 4-\mathrm{C} 5$ & $1.7(4)$ & $\mathrm{C} 14-\mathrm{C} 19-\mathrm{C} 20 \mathrm{~B}-\mathrm{O} 5 \mathrm{~B}$ & $61.9(7)$ \\
\hline $\mathrm{C} 2-\mathrm{C} 3-\mathrm{C} 4-\mathrm{C} 13$ & $-175.6(3)$ & $\mathrm{C} 14-\mathrm{C} 19-\mathrm{C} 20 \mathrm{~B}-\mathrm{O} 6 \mathrm{~B}$ & $-116.7(5)$ \\
\hline $\mathrm{C} 3-\mathrm{C} 4-\mathrm{C} 5-\mathrm{O} 3$ & $-179.9(3)$ & $\mathrm{C} 15-\mathrm{C} 14-\mathrm{C} 19-\mathrm{C} 18$ & $-3.4(3)$ \\
\hline $\mathrm{C} 3-\mathrm{C} 4-\mathrm{C} 5-\mathrm{C} 6$ & $-1.2(4)$ & $\mathrm{C} 15-\mathrm{C} 14-\mathrm{C} 19-\mathrm{C} 20 \mathrm{~A}$ & $-172.9(4)$ \\
\hline
\end{tabular}




$\begin{array}{ll}\mathrm{C} 3-\mathrm{C} 4-\mathrm{C} 13-\mathrm{C} 10 & 178.5(3) \\ \mathrm{C} 3-\mathrm{C} 4-\mathrm{C} 13-\mathrm{C} 14 & 1.5(4) \\ \mathrm{C} 4-\mathrm{C} 5-\mathrm{C} 6-\mathrm{C} 1 & -0.1(5) \\ \mathrm{C} 4-\mathrm{C} 13-\mathrm{C} 14-\mathrm{C} 15 & 72.7(3) \\ \mathrm{C} 4-\mathrm{C} 13-\mathrm{C} 14-\mathrm{C} 19 & -107.4(3) \\ \mathrm{C} 5-\mathrm{O} 3-\mathrm{C} 9-\mathrm{C} 8 & 176.9(3) \\ \mathrm{C} 5-\mathrm{O} 3-\mathrm{C} 9-\mathrm{C} 10 & -1.5(4) \\ \mathrm{C} 5-\mathrm{C} 4-\mathrm{C} 13-\mathrm{C} 10 & 1.3(4) \\ \mathrm{C} 5-\mathrm{C} 4-\mathrm{C} 13-\mathrm{C} 14 & -175.7(2) \\ \mathrm{C} 6-\mathrm{C} 1-\mathrm{C} 2-\mathrm{C} 11 & -179.4(3) \\ \mathrm{C} 6-\mathrm{C} 1-\mathrm{C} 2-\mathrm{C} 3 & -0.7(5) \\ \mathrm{C} 7-\mathrm{C} 8-\mathrm{C} 9-\mathrm{O} 3 & -178.1(3) \\ \mathrm{C} 7-\mathrm{C} 8-\mathrm{C} 9-\mathrm{C} 10 & 0.1(5) \\ \mathrm{C} 7-\mathrm{C} 8-\mathrm{C} 24-\mathrm{C} 25 & -88.3(7) \\ \mathrm{C} 7-\mathrm{C} 8-\mathrm{C} 24 \mathrm{~A}-\mathrm{C} 25 \mathrm{~A} & 94.0(8) \\ \mathrm{C} 8-\mathrm{C} 7-\mathrm{C} 12-\mathrm{C} 2 & 179.0(3) \\ \mathrm{C} 8-\mathrm{C} 7-\mathrm{C} 12-\mathrm{C} 11 & -0.2(5) \\ \mathrm{C} 8-\mathrm{C} 9-\mathrm{C} 10-\mathrm{C} 11 & 0.3(4) \\ \mathrm{C} 8-\mathrm{C} 9-\mathrm{C} 10-\mathrm{C} 13 & -177.9(3) \\ \mathrm{C} 8-\mathrm{C} 24-\mathrm{C} 25-\mathrm{C} 26 & -125(2) \\ \mathrm{C} 8-\mathrm{C} 24 \mathrm{~A}-\mathrm{C} 25 \mathrm{~A}-\mathrm{C} 26 \mathrm{~A} & -126.4(15) \\ \mathrm{C} 9-\mathrm{O} 3-\mathrm{C} 5-\mathrm{C} 4 & 2.6(4) \\ \mathrm{C} 9-\mathrm{O} 3-\mathrm{C} 5-\mathrm{C} 6 & -176.1(3) \\ \mathrm{C} 9-\mathrm{C} 8-\mathrm{C} 24-\mathrm{C} 25 & 101.7(7) \\ \mathrm{C} 9-\mathrm{C} 8-\mathrm{C} 24 \mathrm{~A}-\mathrm{C} 25 \mathrm{~A} & -97.7(8) \\ \mathrm{C} 9-\mathrm{C} 10-\mathrm{C} 11-\mathrm{C} 12 & -0.6(4) \\ & \end{array}$

$\begin{array}{ll}\mathrm{C} 15-\mathrm{C} 14-\mathrm{C} 19-\mathrm{C} 20 \mathrm{~B} & 173.9(4) \\ \mathrm{C} 15-\mathrm{C} 16-\mathrm{C} 17-\mathrm{C} 15 & 177.90(18) \\ \mathrm{C} 15-\mathrm{C} 16-\mathrm{C} 17-\mathrm{C} 18 & -1.4(3) \\ \mathrm{C} 16-\mathrm{C} 17-\mathrm{C} 18-\mathrm{C} 16 & 179.24(18) \\ \mathrm{C} 16-\mathrm{C} 17-\mathrm{C} 18-\mathrm{C} 19 & 2.3(4) \\ \mathrm{C} 17-\mathrm{C} 18-\mathrm{C} 19-\mathrm{C} 14 & 0.1(4) \\ \mathrm{C} 17-\mathrm{C} 18-\mathrm{C} 19-\mathrm{C} 20 \mathrm{~A} & 168.8(4) \\ \mathrm{C} 17-\mathrm{C} 18-\mathrm{C} 19-\mathrm{C} 20 \mathrm{~B} & -177.2(4) \\ \mathrm{C} 18-\mathrm{C} 19-\mathrm{C} 20 \mathrm{~A}-\mathrm{O} 5 \mathrm{~A} & -110.4(6) \\ \mathrm{C} 18-\mathrm{C} 19-\mathrm{C} 20 \mathrm{~A}-\mathrm{O} 6 \mathrm{~A} & 70.7(5) \\ \mathrm{C} 18-\mathrm{C} 19-\mathrm{C} 20 \mathrm{~B}-\mathrm{O} 5 \mathrm{~B} & -120.8(6) \\ \mathrm{C} 18-\mathrm{C} 19-\mathrm{C} 20 \mathrm{~B}-\mathrm{O} 6 \mathrm{~B} & 60.6(6) \\ \mathrm{C} 19-\mathrm{C} 14-\mathrm{C} 15-\mathrm{C} 13 & -174.23(18) \\ \mathrm{C} 19-\mathrm{C} 14-\mathrm{C} 15-\mathrm{C} 16 & 4.4(3) \\ \mathrm{O} 6 \mathrm{~A}-\mathrm{C} 21 \mathrm{~A}-\mathrm{C} 22 \mathrm{~A}-\mathrm{C} 23 \mathrm{~A} & -118.3(10) \\ \mathrm{C} 20 \mathrm{~A}-\mathrm{O} 6 \mathrm{~A}-\mathrm{C} 21 \mathrm{~A}-\mathrm{C} 22 \mathrm{~A} & -88.9(8) \\ \mathrm{C} 21 \mathrm{~A}-\mathrm{O} 6 \mathrm{~A}-\mathrm{C} 20 \mathrm{~A}-\mathrm{C} 19 & -178.2(5) \\ \mathrm{C} 21 \mathrm{~A}-\mathrm{O} 6 \mathrm{~A}-\mathrm{C} 20 \mathrm{~A}-\mathrm{O} 5 \mathrm{~A} & 2.8(10) \\ \mathrm{O} 6 \mathrm{~B}-\mathrm{C} 21 \mathrm{~B}-\mathrm{C} 22 \mathrm{~B}-\mathrm{C} 23 \mathrm{~B} & 107.2(16) \\ \mathrm{C} 20 \mathrm{~B}-\mathrm{O} 6 \mathrm{~B}-\mathrm{C} 21 \mathrm{~B}-\mathrm{C} 22 \mathrm{~B} & -97.9(11) \\ \mathrm{C} 21 \mathrm{~B}-\mathrm{O} 6 \mathrm{~B}-\mathrm{C} 20 \mathrm{~B}-\mathrm{C} 19 & 179.9(5) \\ \mathrm{C} 21 \mathrm{~B}-\mathrm{O} 6 \mathrm{~B}-\mathrm{C} 20 \mathrm{~B}-\mathrm{O} 5 \mathrm{~B} & 1.4(10) \\ \mathrm{C} 24-\mathrm{C} 8-\mathrm{C} 9-\mathrm{O} 3 & -8.2(5) \\ \mathrm{C} 24-\mathrm{C} 8-\mathrm{C} 9-\mathrm{C} 10 & 170.1(4) \\ \mathrm{C} 24 \mathrm{~A}-\mathrm{C} 8-\mathrm{C} 9-\mathrm{O} 3 & 13.8(6) \\ \mathrm{C} 24 \mathrm{~A}-\mathrm{C} 8-\mathrm{C} 9-\mathrm{C} 10 & -167.9(4) \\ & \end{array}$

Hydrogen-bond geometry $\left(A,{ }^{\circ}\right)$

\begin{tabular}{lllll}
\hline$D-\mathrm{H} \cdots A$ & $D-\mathrm{H}$ & $\mathrm{H} \cdots A$ & $D \cdots A$ & $D-\mathrm{H} \cdots A$ \\
\hline $\mathrm{O} 2-\mathrm{H} 2 \cdots \mathrm{O} 4^{\mathrm{i}}$ & $0.74(4)$ & $1.86(4)$ & $2.595(3)$ & $172(4)$ \\
\hline
\end{tabular}

Symmetry code: (i) $y+1 / 4,-x+3 / 4,-z+3 / 4$. 